\title{
Dutch theoreticians win physics prize
}

London

This year's Nobel Prize for Physics has gone to Geradus 't Hooft of the University of Utrecht and Martinus Veltman, emeritus professor at the same university, who showed how the complex mathematical structure of the theory of the electroweak interaction need not be an obstacle to predictive calculations.

They showed how to perform precise calculations of the physical properties of the new particles predicted by this and related theories, many of which have now received experimental confirmation. "The major advances in particle physics of the past 25 years depend on their work," says Christopher Llewellyn-Smith of University College London.

Initial progress with the theory of the electroweak interaction was hampered by the same problem that had plagued the development of quantum electrodynamics (QED) - how to cope mathematically with the short-lived particles and anti-particles that quantum theory predicts will surround

\section{Publishers agree on a 'seamless web'}

London

The publishers of the world's leading scientific journals have agreed to adopt common procedures that will allow a 'seamless web' linking references in the articles they publish to the source papers in their respective publications through the World-Wide Web.

The agreement was reached in Frankfurt on Tuesday by 300 industry executives attending a meeting of the International Association of Science, Technology and Medical Publishers. It is the result of discussions and trials that have been taking place over the past two years.

The purpose of what is described as "the world's largest research web" will be to ensure that any paper cited in the electronic versions of the journals covered - including the Nature family of journals - will be directly accessible via the web.

It is expected that eventually between five and ten million scientific articles and their references will be interconnected in this way. A final plan is to be discussed at a meeting in London in December.

"This simple act begins to deliver on the full promise of the web," says Robert Campbell, chair of the association. "We are working together to serve our primary constituencies: the authors and readers who comprise the world community of scientists."

David Dickson

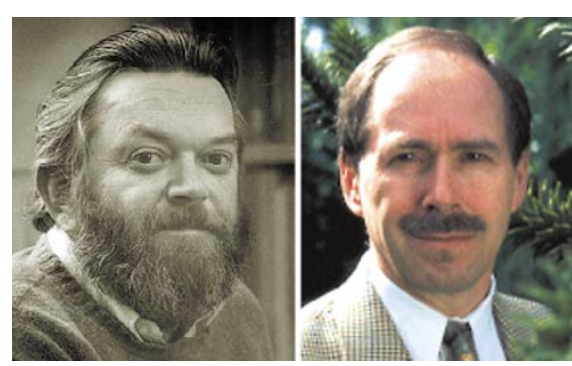

Veltman (left) and 't Hooft: Dutch double success.

a 'naked' central particle.

For QED, the problem was solved by the introduction of a 'renormalization' procedure: the experimentally determined values of mass and charge for the particle are used as 'renormalized' input parameters.

But the electroweak theory does not possess the symmetry simplicity of QED. The particles mediating the electromagnetic interaction are the massless, non-interacting photons; for the electroweak interaction, however, they are massive and interacting. The mathematical difficulties arising from these additional properties were such that many theorists at the time doubted the physical viability of the electroweak theory.

Veltman, optimistic that the calculational difficulties could be overcome, developed the computational tools and showed that many of the more problematic aspects of the electroweak theory effectively cancelled each other out.

According to Llewellyn-Smith, "Veltman was the driving force keeping interest in such theories alive." 't Hooft, Veltman's student at the time, had the insight that if the masses of particles such as $\mathrm{W}$ and $\mathrm{Z}$ were a natural consequence of the theory, rather than input parameters, then the theory became selfconsistent and tractable.

These combined developments were key, and the theory of the electroweak interaction became, by the early 1970 s, a practical — and very successful- theoretical tool for performing precise calculations. Calculations of physical quantities associated with the $\mathrm{W}$ and $\mathrm{Z}$ particles and the top quark show agreement with recent measurements. KarlZiemelis

\section{Split-second chemistry is rewarded}

\section{London}

The Nobel Prize for Chemistry has been awarded to Ahmed Zewail of the California Institute of Technology for his role in developing techniques of ultrafast spectroscopy for studying molecular change in real time.

The fundamental time-scale of chemical reactions is set by the speed at which the reactant molecules pass through the transition state. This involves atomic motions which typically occupy just a few tens of femtoseconds $\left(10^{-15} \mathrm{~s}\right)$.

Short pulses of laser light are used to capture this change. A series of femtosecond pulses provides a strobed sequence of the molecules' transformation. Each pulse records a slice through the absorption spectrum, modified by the change in the molecule.

This process requires, however, that one knows a 'zero' time from when the change began. This involves synchronizing two pulses with femtosecond precision. The initial 'pump' pulse provides the stimulus for a photochemical process - for example, boosting a collection of molecules into an excited state. Subsequent pulses then interrogate the molecules as they pass through the transition state to the products.

Zewail has pioneered the implementation of this scheme. His early experiments focused on the simplest case: unimolecular

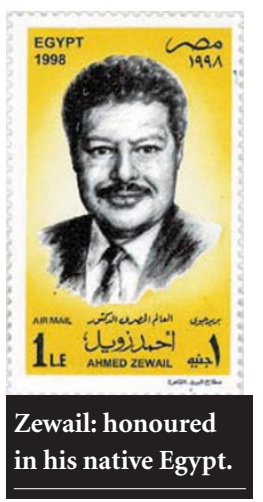

photodissociation, whereby a molecule absorbs a photon and fragments into two. His later work encompasses more complex reactions, such as the dissociation of $\mathrm{C}_{2} \mathrm{I}_{2} \mathrm{~F}_{4}$ into three fragments, bimolecular processes such as the reaction of carbon dioxide with a hydrogen radical, and the influence of solvent cages on reaction dynamics in solution.

John Simons of the University of Oxford says that Zewail has been "marvellously effective in demonstrating the potential of ultrafast laser techniques — of showing quantum behaviour happening before your eyes". These methods are now being applied beyond elementary test cases, for example to elucidate some of the rapid electron transfer processes in photosynthesis.

It seems inevitable that ultrafast change in biological systems will receive increasing attention. Another goal is the use of pulsed laser techniques for ultrafast diffraction, so that precise changes in atomic coordinates can be tracked during chemical change.

Zewail has been a favourite for the award for several years.

Philip Ball 